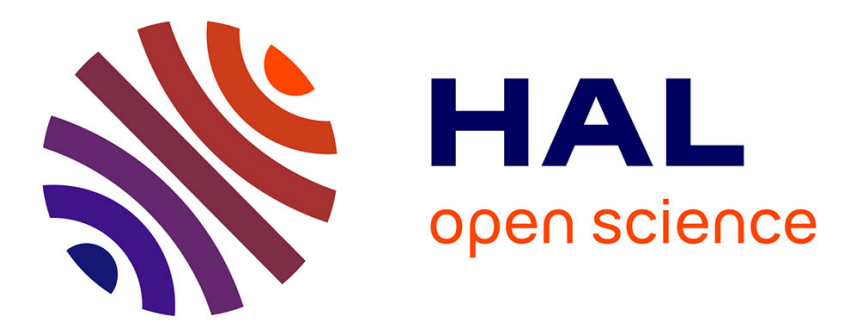

\title{
Atom-to-ion transformation time in singly charged ECRISs
}

\author{
P. Jardin, W. Farabolini, G. Gaubert, J.Y. Pacquet, J. Cornell, F. Durantel, \\ C. Huet-Equilbec, N. Lecesne, R. Leroy, M.G. Saint-Laurent, et al.
}

\section{- To cite this version:}

P. Jardin, W. Farabolini, G. Gaubert, J.Y. Pacquet, J. Cornell, et al.. Atom-to-ion transformation time in singly charged ECRISs. Nuclear Instruments and Methods in Physics Research Section B: Beam Interactions with Materials and Atoms, 2004, 225, pp.374-382. 10.1016/j.nimb.2004.06.004 . in2p3-00022189

\section{HAL Id: in2p3-00022189 https://hal.in2p3.fr/in2p3-00022189}

Submitted on 1 Sep 2004

HAL is a multi-disciplinary open access archive for the deposit and dissemination of scientific research documents, whether they are published or not. The documents may come from teaching and research institutions in France or abroad, or from public or private research centers.
L'archive ouverte pluridisciplinaire HAL, est destinée au dépôt et à la diffusion de documents scientifiques de niveau recherche, publiés ou non, émanant des établissements d'enseignement et de recherche français ou étrangers, des laboratoires publics ou privés. 


\title{
Atom-to-ion transformation time in singly-charged ECRISs
}

P. Jardin ${ }^{\text {a) }}$, W. Farabolini, G. Gaubert, J.Y. Pacquet, J. Cornell, F. Durantel, C. Huet-Equilbec, N. Lecesne, R. Leroy, M.G. Saint Laurent, C. Barué, C. Canet, M. Dubois, M. Dupuis, J-L. Flambard, P. Lehérissier, F. Lemagnen, O. Tuske, A.C.C Villari.

GANIL (Grand Accélérateur National d'Ions Lourds). Bd Henri Becquerel. BP 55027. F14076 CAEN cedex 5

\begin{abstract}
We report a series of measurements performed to characterize the atom-to-ion transformation time in singly-charged Electron Cyclotron Resonance Ion Sources (ECRISs). Three ECRISs were used with $\mathrm{He}, \mathrm{Ne}, \mathrm{Ar}$ and $\mathrm{Kr}$ noble gases. The transformation time as a function of the support gas flow, the ionization efficiency, and source volume is presented and compared to a calculation made with a simple dynamic model.

\section{PACS codes :}

Keywords : Electron cyclotron resonance ion source; Isotope separation on line ; atom to ion transformation time ; Support gas ; Singly-charged ion
\end{abstract}

\section{Introduction}

Since the beginning of radioactive ion production by the ISOL (Isotope Separation On Line) method, there have been attempts to design a Target/Ion-Source System (TISS), in which the transformation of radioactive atoms is faster than the radioactive decay. Moreover, every time that the transformation speed is increased, one tries to reach another more exotic isotope, closer to the "drip line", and thus with a shorter half-life.

Since the production cross-sections are defined by the choice of the target-projectile pair, the production of radioactive fragments in a thick target can be computed before the experiment ${ }^{1}$. The remaining problem is transforming the radioactive atoms into ions within a time much shorter than the half-life.

One has then to deal with physical and chemical features of each atomic specie; these properties cause atoms to have different behavior during diffusion out of the target, effusion into the TISS and during ionization. For the $1+/ \mathrm{n}+$ project $^{2,3}$ and the SPIRAL II proposal ${ }^{4}$, new targets and ionization sources have to be designed at GANIL ${ }^{5}$.

Owing to their ionization efficiencies with stable elements, their simplicity and their low cost, an ECRIS (Electron Cyclotron Resonance Ion Source) that is producing ions mainly in charge state $1+$ seems to be well suited to radioactive ion production of noble gases. A series of measurements has been carried out with these ECRISs to improve our knowledge of the atomto-ion transformation time, and to apply the results of these observations to the future on-line radioactive ion production systems ${ }^{6}$. Since the ionization efficiencies for the $\mathrm{n}+$ charge states $(\mathrm{n} \geq 1)$ decreases rapidly with $\mathrm{n}$ (e.g. less than $7 \%$ for $\mathrm{Ar}^{2+}$ and less than $1 \%$ for $\mathrm{Ar}^{3+}$ ), the fraction of atoms ionized in a multi-charged state is neglected in the following text.

a) corresponding author. Tel.:+33-2-3145-4659; Fax: +33-2-3145-4728.

E-mail address: jardin@ganil.fr 
After describing the set-up, we first report on measurements performed off-line with stable atoms in order to characterize the atom-to-ion transformation time, the effusion time and the influence of the volume. A simple model is presented and an experimental tool is given to evaluate the reliability of such a measurement.

\section{Description of the setup}

As previously described ${ }^{7}$, the setup includes an ECRIS ion source with its radio frequency (RF) emitter, its support gas feed and a calibrated leak (Fig. 1). Three 2.45-GHz ECRIS ion sources of different volumes were used: MINIMONO ${ }^{8}$, GDI5 and MONO1000,10.

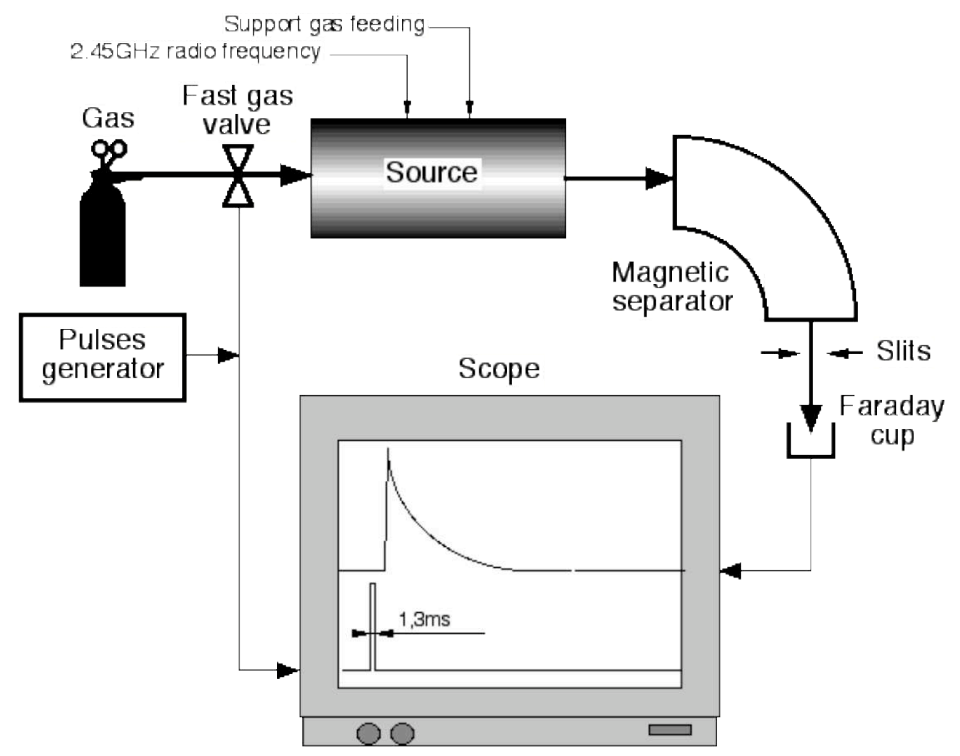

Fig. 1: Experimental setup

Two gases are continuously injected into the source: the support gas, necessary to ignite and sustain the plasma, and the gas of interest injected through a calibrated leak, necessary to measure the ionization efficiency by observing the ratio between the calibrated leak and the corresponding current measured on the Faraday cup (after correction for the line transport efficiency).

The same gas as that injected into the leak is also injected in short pulses $(1.3 \mathrm{~ms})$ into the source through a fast valve. The electric signal delivered to the valve triggers a scope on which the signal collected on the Faraday cup is recorded. The transformation time of atoms into ions $T_{\mathrm{ai}}$ is measured for different proportions $(Q=10 \%, 50 \%, 90 \%)$ of ions produced by the gas pulse, which have left the source.

For a given setting of all the working parameters, the reproducibility of the ion pulses is very good, in height (variation below 10\%) and in shape. To reduce the noise, several successive pulses were averaged. The number of pulses, between 2 and 16, depended on the ratio between the noise and the pulse height. The interval between two pulses was tuned to avoid pile-up.

\section{Plasma stability and ionization efficiency error}

In order to compare different measurements, it is important to make sure of the plasma stability and to limit the efficiency error. The time measurements were made only after obtaining a stable regime of the plasma. This was controlled through the current delivered by the high-voltage supply. The measurement accuracy of this current is $\sim 1 \mu \mathrm{A}$, to be compared to the measured currents from $100 \mu \mathrm{A}$ up to $1 \mathrm{~mA}$. The stability was better than $1 \%$. 
Nevertheless, each response time was measured over several minutes. However, we did not try to maintain the same working conditions of the source during the measurement over many hours. This would have required accurate control of parameters like the room temperature, since variations can change the value of the calibrated leak, the aperture of the support gas valve and the pressure of gas in the feed-bottles. Each measurement required a new setting of the RF power. Although the RF power was kept low to shorten the delay needed to obtain a thermal equilibrium and a stable out-gazing in the source chamber, variations in the efficiency are probably inevitable. For these numerous reasons of uncertainty, the ionization efficiencies are given within error bars of $20 \%$.

\section{Experimental operation}

In a continuous regime, i.e. without gas pulses, a measurement of the ionization efficiency of He versus the support gas flow shows that the ionization efficiency of the source is very sensitive to the gas density (Fig. 2) as is commonly observed.

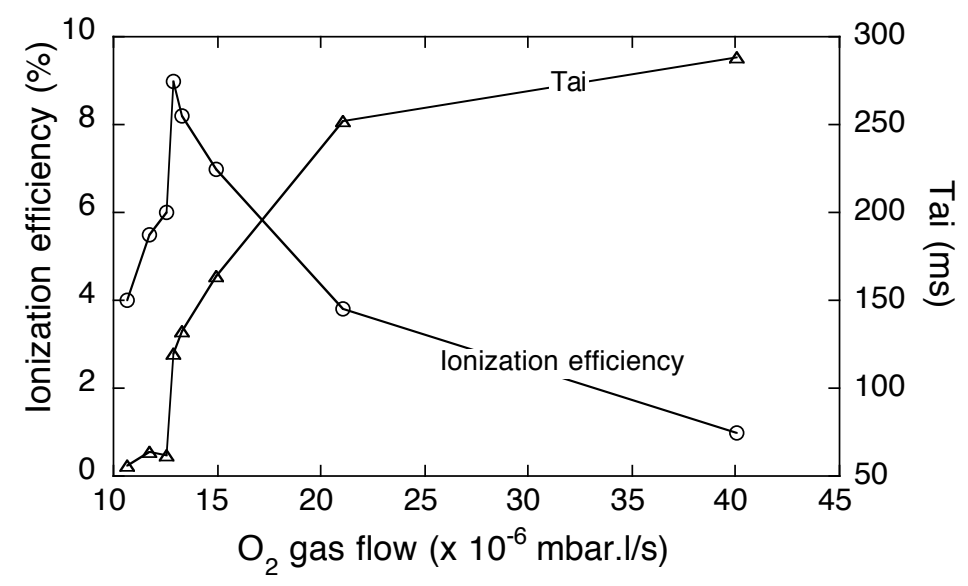

Fig 2: Ionization efficiency of He to $\mathrm{He}^{+}$(left-hand scale and circles) and atom-to-ion transformation time $T_{a i}$ (right-hand scale and triangles) versus the $\mathrm{O}_{2}$ pressure, observed with the MINIMONO source.

At the maximum of the observed efficiency, a small variation of gas flow leads to a large change in the ionization efficiency. For example, a decrease of $2 \times 10^{-6} \mathrm{mbar} .1 / \mathrm{s}$ in the gas flow leads to a reduction of the ionization efficiency by a factor of 2 . In order to avoid a significant pressure variation during the pulse injection, and thus a variation of the ionization efficiency, a small flow of gas is required in the pulse compared to the gas coming from the calibrated leak (some particle $\mu \mathrm{A}$, or $\mu \mathrm{Ap}$ ), which is still very low compared to the support gas (some $100 \mu \mathrm{Ap}$ ). In the following, tests were performed with different gases: $\mathrm{He}, \mathrm{Ne}, \mathrm{Ar}$ and $\mathrm{Kr}$. Whenever possible, the level of the gas pulse was kept low so as to induce negligible perturbation of the source.

\section{Results}

The transformation time $T_{\mathrm{ai}}$ versus the ionization efficiency are presented for two sources (Figs. 3 and 4). In both cases, the ionization efficiency was adjusted using the support gas flow. 


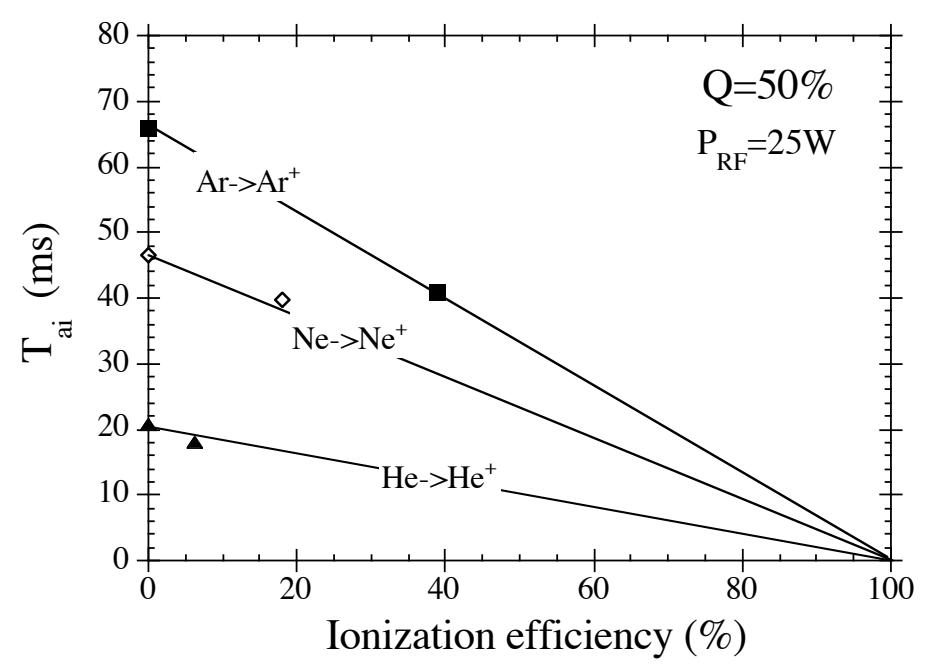

Fig 3: Time $T_{a i}$ for the transformation of atoms into ions in MINIMONO versus ionization efficiency for pulses of He, Ne and Ar gases. For ionization efficiencies close to 0, the time $T_{a i}$ corresponds to a pure effusion time. The meaning of the lines is explained paragraph VI.a. relation (13).

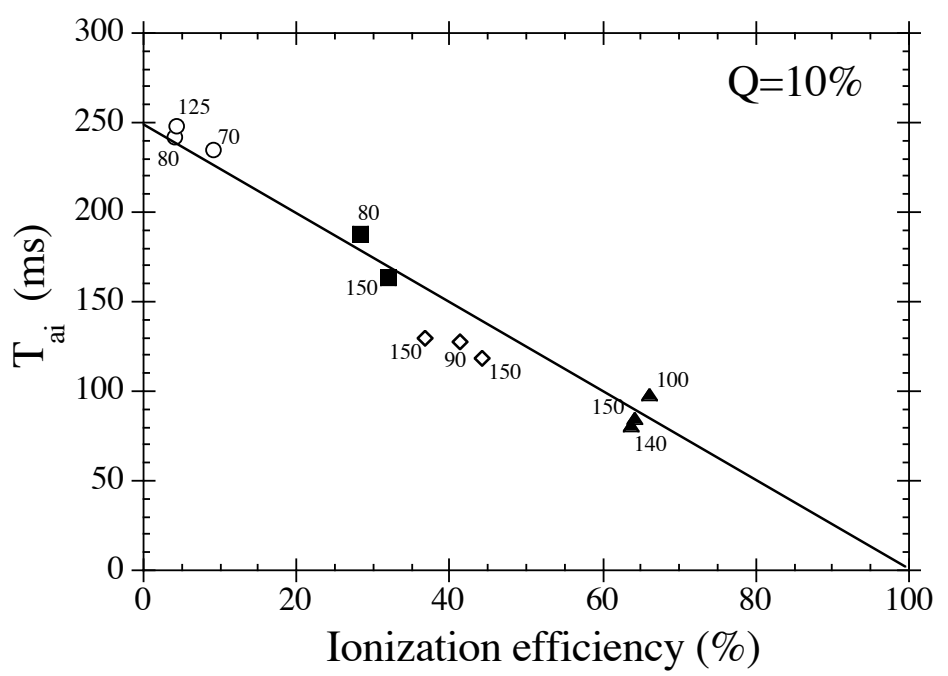

Fig 4: Time $T_{a i}$ versus ionization efficiency for pulses of Ar. The same symbols correspond to a same flow of support gas. For each measurement, the $R F$ power is given.

The first source was a MINIMONO ECRIS. The volume of the chamber was equal to $117 \mathrm{~cm}^{3}$. Its extraction hole was $4 \mathrm{~mm}$ in diameter. For both sources, the time $T_{\text {ai }}$ increases as the efficiency decreases. For ionization efficiencies very close to 0 , the measured times $T_{\mathrm{ai}}$ in Fig. 3 correspond to pure effusion times, which can be calculated from equations (2) and (13) given in the next chapter.

The second source is also a 2.45-GHz ECRIS, GDI5, based on the same magnetic principle. The main difference is the chamber volume, which is of the order of $6000 \mathrm{~cm}^{3}$. The extraction hole is $7 \mathrm{~mm}$ in diameter. The time $T_{\text {ai }}$ is determined for $Q=10 \%$ and for the $\mathrm{Ar}$ to $\mathrm{Ar}^{+}$ transformation. The results are reported in figure 4. Each symbol used corresponds to a particular support gas flow. We note that for a given gas flow, a variation in the RF power of the order of $50 \%$ has only a weak effect on the ionization efficiency. From the measurements made for ionization efficiencies in the range of $4 \%$ to $65 \%$, one can deduce that for an 
ionization efficiency equal to zero, the time $T_{\mathrm{ai}}$ is close to $250 \mathrm{~ms}$, which corresponds to the pure effusion time. Unfortunately, no measurement of $T_{\text {ai }}$ was made for ionization efficiency very close to zero.

To have a global view of this behavior, we built a simple model.

\section{Model and comparison with the measurements}

VI.a. Atom-to-ion transformation time

The removal of atoms from the source is due to the transformation of the atoms into ions (ionization process) and to losses through the extraction hole of neutral atoms leaving the ion source before being ionized (effusion).

If a number $x_{a 0}$ of atoms contained in a pulse are injected into the source at time $t=0$, and if the ionization efficiency is close to 0 , the number $x_{a}$ of atoms in the source at $t$ will be given by:

$$
x_{a}(t)=x_{a 0} \cdot e^{-\lambda \text { eff } t} \text { (1) with } \lambda \text { eff }=C / V \text {. }
$$

$C$ is the conductance of the extraction hole of the source and $V$ its volume. If one considers that the vacuum chamber has a high volume-to-surface ratio, the effusion law will be close to an exponential.

Since the ionization of the atoms by the plasma contributes to the removal of atoms, one has to estimate the number $d x_{a}$ of atoms transformed into ions per unit volume and unit time $d r^{3} d t$ by collisions with the charged particles of the plasma. In this first approach we consider only the ionization by electron impact. Thus $d x_{a}$ can be estimated through this expression

$$
\frac{d x_{a}}{d r^{3} \cdot d t}=\int_{0}^{\infty} \sigma\left(v_{e}\right) \cdot v_{e} \cdot n_{e} \cdot \frac{x_{a}}{V} \cdot f\left(v_{e}\right) \cdot d v_{e}
$$

where $v_{e}$ is the velocity of the electrons, $\sigma$ is the ionization cross section by electron impact, $n_{e}$ and $\frac{x_{a}}{V}$ are respectively the electron and the atom densities. $f\left(v_{e}\right)$ is the velocity distribution of the electrons such as

$$
\int_{0}^{\infty} f\left(v_{e}\right) d v_{e}=1
$$

The velocity of the atoms is neglected in comparison with the electronic velocity. If we ignore the dependence of $f\left(v_{e}\right)$ and $n_{e}$ on their location in the source, the integration of the expression (3) consists in multiplying it by a volume $V_{p}$, which corresponds to an average value of the plasma volume.

$$
\frac{d x_{a}}{d t}=\frac{V_{p}}{V} \cdot\left\langle\sigma \cdot v_{e}\right\rangle \cdot n_{e} \cdot x_{a}
$$

Where

$$
\left\langle\sigma \cdot v_{e}\right\rangle=\int_{0}^{\infty} \sigma\left(v_{e}\right) \cdot v_{e} \cdot f\left(v_{e}\right) \cdot d v_{e}
$$

The disappearance of the atoms due to ionization is thus governed by the following equation: 


$$
x_{a}(t)=x_{a 0} e^{-\lambda_{\text {ion. } . t}}(6) \quad \text { with } \quad \lambda_{\text {ion }}=\left\langle\sigma \cdot v_{e}\right\rangle \cdot \frac{V_{p}}{V} \cdot n_{e} .
$$

To maximize $\lambda_{\text {ion }}$, it is thus convenient to have the plasma volume as close as possible to the source volume in order to avoid a dead volume where atoms cannot be ionized, and obviously to maximize the electron density.

We now consider the removal of atoms from the source by ionization and effusion:

We obtain the following integrated form:

$$
\frac{d x_{a}}{d t}=-\left\langle\sigma \cdot v_{e}\right\rangle n_{e} \frac{V_{p}}{V} x_{a}-\frac{C}{V} x_{a} .
$$

$$
x_{a}(t)=x_{a 0} \cdot e^{-\left(\lambda_{\text {ion }}+\lambda_{\text {eff }}\right) \cdot t}=x_{a 0} \cdot e^{-\lambda_{\text {tot. } t}} .
$$

The number $x_{i}$ of ions created per time unit is given by:

$$
\frac{d x_{i}}{d t}=\lambda_{\text {ion }} \cdot x_{a}
$$

From this, we deduce:

$$
x_{i}=\frac{\lambda_{\text {ion }}}{\lambda_{\text {ion }}+\lambda_{\text {eff }}} \cdot x_{a 0} \cdot\left(1-e^{-\left(\lambda_{i o n}+\lambda_{e f f}\right) . t}\right),
$$

considering that $x_{i}=0$ at $t=0$, i.e. when the pulse of atoms is injected. The ionization efficiency $\varepsilon i$ corresponds to the ratio $x_{i} / x_{a 0}$ when $t \rightarrow \infty$, and thus

$$
\varepsilon_{i}=\frac{\lambda_{\text {ion }}}{\lambda_{\text {ion }}+\lambda_{\text {eff }}} \quad \text { (11) } \quad \text { and } \lambda_{\text {ion }}=\frac{\varepsilon_{i}}{1-\varepsilon_{i}} \lambda_{\text {eff }} \text {. }
$$

This expression is still valid in the case of a continuous injection of atoms into the source. Thus a measurement of the ionization efficiency by using a calibrated leak can be sufficient to know the time $T_{\text {ai }}$, if the only route for effusion is via the extraction hole. The time $T_{a i}(Q)$ $(0 \leq Q \leq 1)$ after which a fraction $Q$ of the ions have left the source can be written as:

$$
T_{a i}(Q)=\frac{1}{\lambda_{\text {tot }}} \operatorname{Ln}\left(\frac{1}{1-Q}\right)=\left(1-\varepsilon_{i}\right) \cdot T_{a i}^{\text {eff }}(Q),
$$

where $T_{a i}^{e f f}(Q)$ is the time delay after which a fraction $Q$ of the atoms injected by the pulse would have left the source if only the effusion process were considered (i.e. no ionization). Numerical application of the formula (13) to the MINIMONO and GDI5 sources give the straight lines plotted respectively in Figs. 3 and 4. Good agreement is observed with the experimental data.

Nevertheless, this expression does not take into account the confinement time of the ions inside the plasma. It should be possible to neglect this time in comparison with the effusion time, owing to the temperature of the ions inside the plasma, which is higher than the temperature of the neutral atoms ${ }^{11}$. Thus the ions will reach the exit from the source more quickly, if the ion effusion in the cone of losses can be compared to an atomic effusion and if the ion velocity distribution is assumed to be Maxwellian ${ }^{12}$. More quantitatively, in the case of injecting ${ }^{39} \mathrm{~K}^{+}$ions into a "Phoenix charge breeder ${ }^{13}$ ", the confinement time between injection and exit of the multi-charged ${ }^{39} \mathrm{~K}^{\mathrm{n}+}$ ions inside the plasma was reported to be dependant on the charge state $n$ as given by:

$$
T_{\text {confinement }}(m s) \approx 8.6 \cdot n-25 \text { for } 4 \leq n \leq 11 \text {. (14 }
$$

This suggests a time of the order of some millisecond for the confinement of the ${ }^{39} \mathrm{~K}^{+}$inside the plasma, especially if the source is a singly-charged ion source and therefore requires lower magnetic confinement. 


\section{VI.b. Electronic and atomic densities}

The electron density can be roughly deduced from the volume $V_{P}$ of the plasma ${ }^{14}$ and from $\lambda_{\text {ion }}$ using the relation (7). In the case of single ionization of $\mathrm{Ar}$, in the conditions given in paragraph VII, $\lambda_{i o n} \approx 4.3 s^{-1}$. The mean value $\left\langle\sigma . v_{e}\right\rangle$ is computed multiplying the single ionization cross section of argon by electron impact ${ }^{15}$ by $f\left(v_{e}\right) \cdot v_{e}$, assuming that $f\left(v_{e}\right)$ is a maxwellian distribution. The mean energy of the distribution was close to $16 \mathrm{eV}$. It was determined by measuring the ionization efficiencies of $\mathrm{He}, \mathrm{Ne}$ and Ar mixed in one plasma. The gases being exposed to the same electronic velocity distribution, and knowing the respective ionization cross sections by electron impact for each gas, it has been possible to deduce the electronic velocity distribution and thus the electronic density, which is of the order of $3 \times 10^{11} \mathrm{~cm}^{-3}$ (for an RF power of $50 \mathrm{~W}$ ).

In the usual working conditions, the pressure in an ECRIS corresponds to a molecular regime, for which the interaction between the atoms or molecules is rare and thus cannot justify a uniform atomic density over the volume, as supposed in the viscous regime. In those conditions, the atomic density depends on the emitting law of the atoms after the contact with the wall of the vacuum chamber. Ignoring the form of this law, the atomic density has been supposed uniform.

\section{VI.c. Transformation time versus the source chamber volume}

In a previous paper (ref. 7), measurements of the time $T_{\text {ai }}$ versus the volume of the source chamber were presented. The MINIMONO volume was modified by connecting different additional volumes to the source, using large apertures between them. We now propose to fit these results with an expression deduced from the model. From equations (2), (7) and (13), we obtain:

$$
T_{a i}(Q)=\varepsilon \sqrt{M} \cdot V^{k}+\frac{V}{C\left[\left\langle\sigma \cdot v_{e}\right\rangle \frac{V_{p}}{C} n_{e}+1\right]} \operatorname{Ln}\left(\frac{1}{1-Q}\right) .
$$

Up to now, we considered that the atoms of the pulse fill up the volume instantaneously, leading to a uniform atomic density just after the pulse injection. With respect to the expression (8), at $t=0, x_{a}(t)$ was equal to $x_{a 0}$ and then decreased exponentially. This leads to a linear relation between $T_{a i}(Q)$ and $V$. In fact, a time of some milliseconds is necessary to the atoms to fill up the chamber and the additional volumes. A term $\varepsilon \sqrt{M} \cdot V^{k}$ has been added to take the filling time into account. Its form has no other justification than the experimental evolution of $T_{a i}(Q)$ with $V$. It must increase with $V$, and be proportional to the square root of the gas mass $M . \varepsilon$ and $k$ depend only on $Q$. Previous measurements (Fig. 5) in the case of the $\mathrm{He}, \mathrm{Ne}, \mathrm{Ar}$ and $\mathrm{Kr}$ gases have been fitted by using expression (15). The value of $\left\langle\sigma . v_{e}\right\rangle V_{P} n_{e}$ was estimated and fixed to $292 \mathrm{~cm}^{3} / \mathrm{s}$, using the values of $\lambda_{i o n}$ deduced from the measurements $\left(\lambda_{\text {ion. }} V=\left\langle\sigma . v_{e}\right\rangle V_{P} n_{e}, V=117 \mathrm{~cm}^{3}\right)$. 


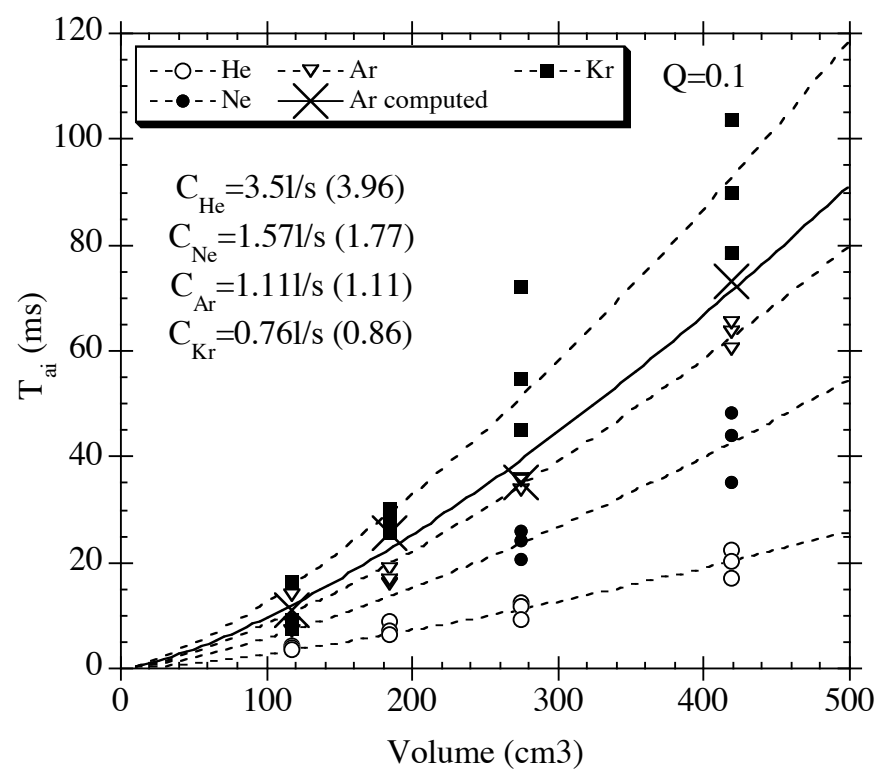

Fig. 5: Transformation time $T_{a i}$ versus the volume of the MINIMONO source for He, $\mathrm{Ne}, \mathrm{Ar}$ and $\mathrm{Kr}$. The dotted lines correspond to fits to the measurements with the relation (15).The bold line and crosses correspond to fit to the Monte-Carlo calculation. For a given gas and volume, a group of three dots corresponds to measurements of the time made for $Q=0.1,0.5$ and 0.9, and transformed assuming the response exponential. The time dispersion of a group of dots reflects the quality of this assumption (see paragraph VI.e).

The values of the conductances have been adjusted to give the best fit for the different gases while respecting the ratios between the conductances, according to the square root of the gas masses. They are slightly lower than the values given in brackets in Fig. 5, which were calculated considering the extraction hole area, the mass of the gas and a temperature of $300 \mathrm{~K}$. The experimental values are deduced from the time measurements, which are lengthened by the complex shapes of the additional volumes and lead to lower apparent conductances. $k$ and $\varepsilon$ were the only free parameters of the fits made with the relation (15). For $k$ equal to 1.4 and $\varepsilon$ equal to $6.7 \times 10^{-2} \mathrm{~s}_{\mathrm{kg}} \mathrm{kg}^{-0.5} \cdot \mathrm{cm}^{-4.2}$, the relation (15) shows a satisfying agreement with the measurements, particularly for $\mathrm{He}$ and Ar.

\section{VI.d. Influence of the pulse height on the response time}

As observed before, the ionization efficiency is sensitive to the gas density present in the source. If the gas pulse modifies the gas density, the ionization efficiency is modified during the pulse and the response time will not correspond to the response time deduced from the ionization efficiency measured in case of a continuous regime. The consequence for the radioactive ion production efficiency can be dramatic, especially for short-lived elements.

To estimate the effect of the gas pulse, we recorded the response shape versus the height of the gas pulse in the case of the MONO1000 source (see Fig. 6). 


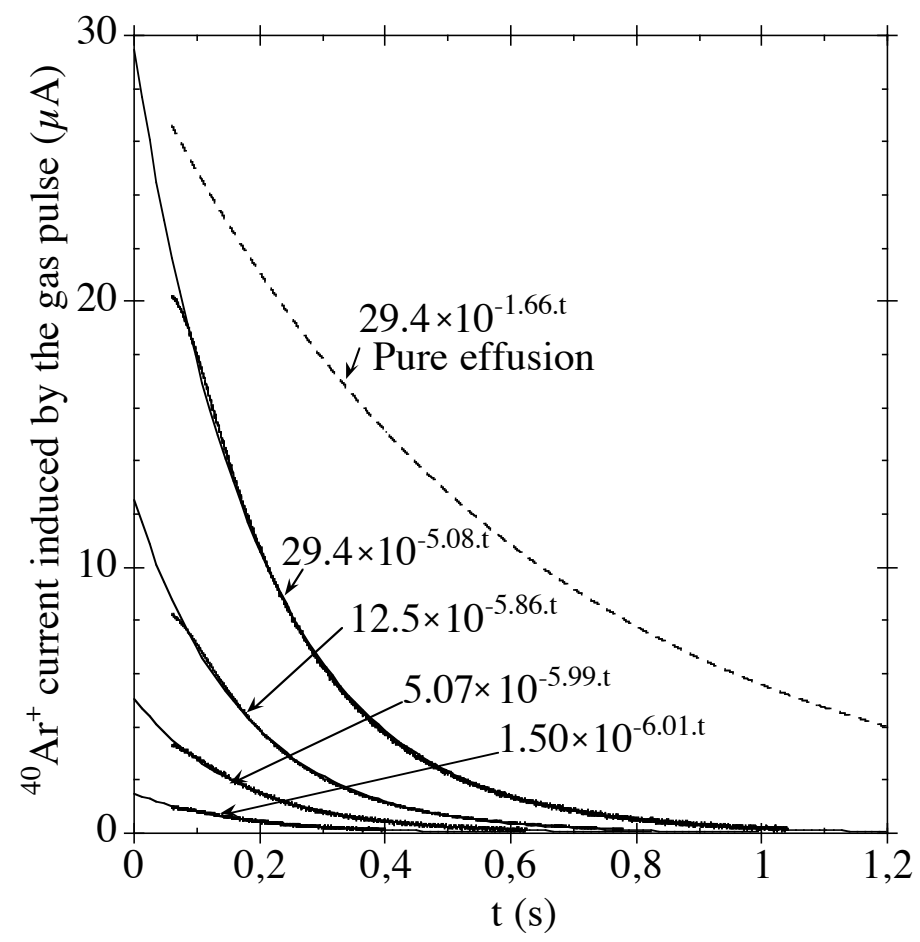

Fig. 6: Measurement of the time response of the ${ }^{40} \mathrm{Ar}$ to ${ }^{40} \mathrm{Ar}$ transformation in the MONO1000 source for different heights of the gas pulse. The shapes of the fits are exponentials. For comparison, the theoretical shape of a pure effusion process is indicated. The heights at $t=0$ and the exponents ( $\lambda$ tot, see text) are deduced from the fits .

17.5 $\mu \mathrm{Ap}$ of ${ }^{40} \mathrm{Ar}$ were injected through the calibrated leak, while pulses of ${ }^{40} \mathrm{Ar}$ varying from $1.5 \mu \mathrm{A}$ to $29.5 \mu \mathrm{A}$ (peak ion current values) were injected through the fast valve. The continuous signal corresponding to the calibrated leak has been subtracted. The beginning of the time response corresponding to the filling of the source by the gas of the pulse has been masked off, to show only the decreasing part of the signal. The ionization efficiency deduced from the continuous component of the current of ${ }^{40} \mathrm{Ar}^{+}$was equal to $69 \%$. The volume of the source and the diameter of the plasma electrode hole were respectively $2310 \mathrm{~cm}^{3}$ and $7 \mathrm{~mm}$.

In the case of Fig. 6, the perturbation is small enough to leave the shape as exponential, even if the exponential decreases slightly faster for low pulses than for higher pulses. In this range of Ar pulse height, one can conclude that the plasma features, contained in the term $\lambda_{\text {ion }}$ (equation 7), are not perturbed by the gas pulse, even for $3 \times 10^{13}$ particles injected (this particle flux is of the order of the maximum radioactive atom flux evaluated for the SPIRAL II project).

VI.e. Influence of the plasma perturbation induced by the gas pulse on the response time In case of radioactive ion production by ISOL method, one has also to take into account the out-gassing of the target induced by the primary beam heating, which can be of the order of $10^{16} \mathrm{pps}$. If this gas flux disturbs the plasma and if the primary beam is continuous, one can retune the plasma on-line towards the optimum working point, if possible. A more complex problem can occur in case of a low frequency (below a few $\mathrm{Hz}$ ) pulsed primary beam and thus a pulsed out-gassing which will induce an oscillation of the working region of the plasma around its optimum. 
If the gas quantity injected during the pulse induces a modification of the plasma features, the time response shape differs from an exponential, as shown Fig. 7.

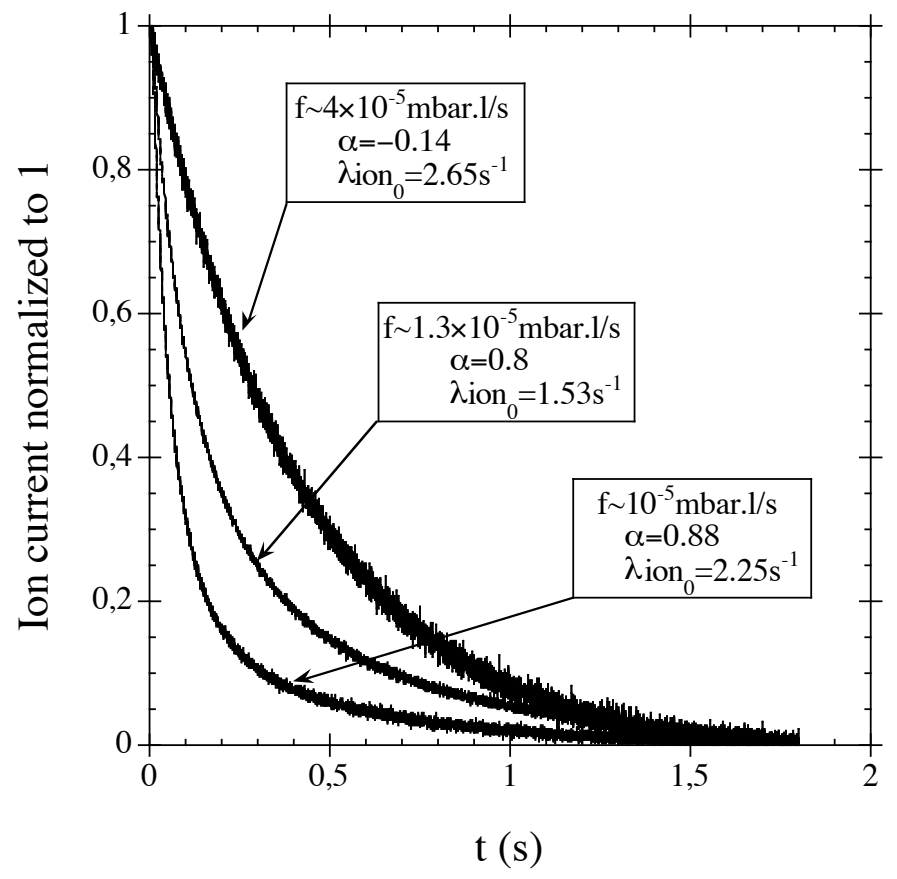

Fig. 7: Measurement of the time response of the ${ }^{4} \mathrm{He}$ to ${ }^{4} \mathrm{He}^{+}$transformation in the MINIMONO source for different flow (f) of support gas. The fits according to the relation (17) are merged with the experimental data.

The resulting shape depends on the pressure region scanned by the pulse. When the gas is injected into the source in which the gas needed for maximum ionization efficiency is missing (left part of the Fig. 2), the gas pulse injection improves the ionization efficiency. Thus at first the pulse decreases quickly. When the pulse gas disappears, the slope becomes smaller until the continuous regime is reached again, as shown in Fig. 7. If the gas is injected in the source when the pressure is too high for the maximum ionization efficiency (right part of the Fig. 2), the effect is less sensitive, the dependence of the efficiency on the pressure being smaller in this pressure region.

If one assumes that the dependence between the gas density and the electron density is linear over the gas density range scanned by the pulse,

$$
n_{e}\left(x_{a}\right)=n_{e 0}+\alpha \cdot x_{a}
$$

the shape of the ion pulse becomes:

$$
x_{a}(t)=x_{a 0} \frac{1-\alpha}{e^{\lambda_{i o n_{0}} . t}-\alpha}, \quad \text { (17) where } \quad \lambda_{i o n_{0}}=\left\langle\sigma \cdot v_{e}\right\rangle \frac{V_{p}}{V} \cdot n_{e 0} .
$$

In case of measurements made with ${ }^{4} \mathrm{He}$ injected in the MINIMONO ECRIS for very different support gas flow, this shape is in a very good agreement with the experiments.

\section{VI.f. Synthesis}

To describe completely the ion current response of an ECR ion source in which a short pulse of noble gas is introduced, the expression (17) has been multiplied by an exponential expression, which represents the filling up of the source: 


$$
x_{a}(t)=x_{a 0} \frac{1-\alpha}{e^{\lambda_{i o n_{0}} t}-\alpha} \cdot\left(1-e^{-\lambda_{i n j} . t}\right)
$$

where $\lambda_{i n j}$ is the characteristic speed of the filling up. An example of a typical response time measurement has been fitted using this expression (Fig. 8).

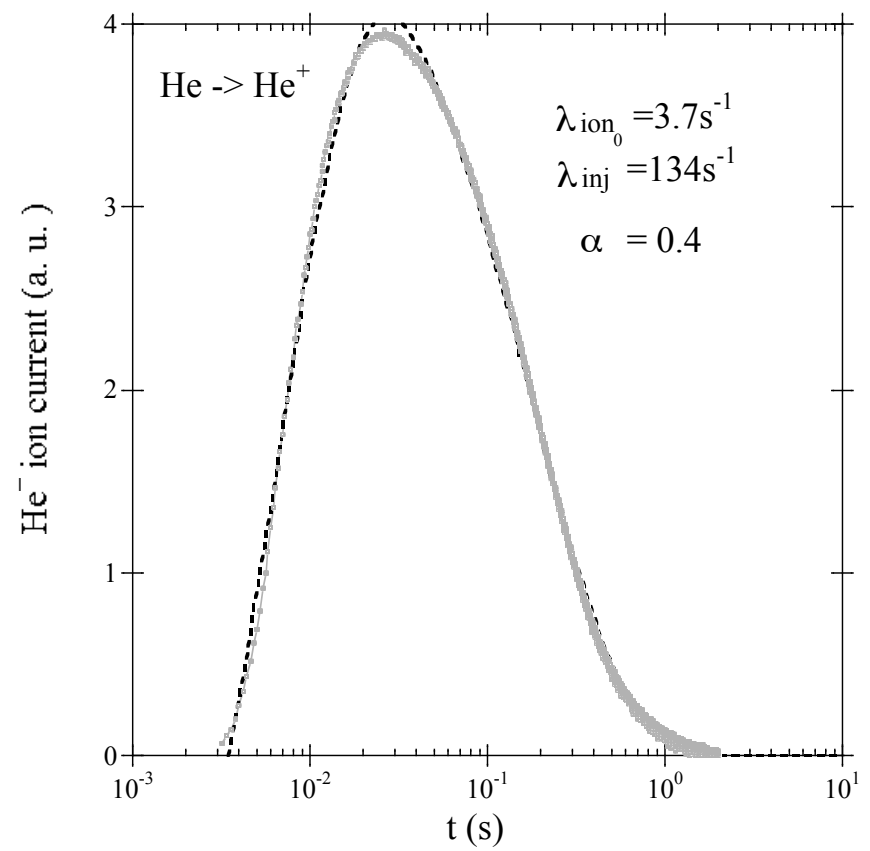

Fig. 8: Example of ion current versus time in the MINIMONO source and the fit (dotted line) to the observed data.

The agreement is very good over the whole time range.

\section{Conclusion}

With simple considerations on the geometry and on the plasma features of singly-charged ECR ion sources, it has been possible to give a description of the source behavior with which one can easily and precisely evaluate the atom-to-ion time transformation, using the shape of the time response.

If exponential, the shape of the time response indicates the possibility of determining the atom-to-ion transformation time by measuring the ionization efficiency in a continuous regime with the usual method of a calibrated leak.

If not exponential, the shape gives an interesting tool to evaluate the sensitivity of the source to a gas perturbation, and thus to evaluate its ability to conserve - during radioactive ion production experiments - the ionization efficiency measured during off-line experiments.

The study of the time response shape is especially interesting if the gas flow coming from the production target is not negligible compared to the "support" gas flow injected to sustain the plasma. In that case, the working point regime will be shifted. This effect can be eventually balanced by reducing the support gas. The worst case corresponds to a time-varying gas injection, such as that produced by a primary beam pulsed at low frequency. The plasma regime then oscillates at the same frequency around the optimum, in regions where the efficiency can strongly differ from the maximum.

All these results have been taken into account in the design of the $1+$ ECRIS $^{16}$ for radioactive ion production for the SPIRAL II project. Particular attention has been devoted to making the shape of the chamber as close as practically possible to that of the plasma volume $V_{P}$, and 
also to maximizing the plasma ionization, i.e. $\left\langle\sigma \cdot v_{e}\right\rangle n_{e}$ by optimizing the RF injection system. Reducing the volume as suggested will clearly improve the efficiency of the source by minimizing the time spent by the atoms out of the plasma region. We also propose in future to measure the ionization efficiency when the target is at its working temperature (and also eventually when the primary beam is on the production target) while varying the support gas flow, in order to balance the effect on the plasma of the gas desorbed by the target. This should then enable us to control the atom-to-ion transformation time on line in future production runs.

\section{Acknowledgement}

This work was supported by the European contract HPRI CT 199950003

${ }^{1}$ A.C.C. Villari, SIGMASIRa GANIL Programme, based on Summerer formula.

${ }^{2}$ R. Geller, C. Tamburella, J.L. Belmont, Rev. Sci. Instrum. 67 (1996) 1281.

${ }^{3}$ C. Tamburella, J.L. Belmont, G. Bizouard, J.F. Bruandet, R. Geller, G. Gimond and B. Vignon, Rev. Sci. Instrum. 68 (1997) 2319.

${ }^{4}$ SPIRAL II project, see web site of GANIL <http://www.ganil.fr $>$.

${ }^{5}$ R. Leroy in : Proc. ICIS03, 10th International Conference on Ion Sources (Dubna, Russia, 2003), RSI, submitted.

${ }^{6}$ P. Jardin et al. in : Proc. ICIS03, 10th International Conference on Ion Sources (Dubna, Russia, 2003), RSI, accepted for publication.

${ }^{7}$ P. Jardin et al., NIM B 204 (2003) 377.

${ }^{8}$ G. Gaubert et al., Rev. Sci. Instrum. 74 (2003) 956.

${ }^{9}$ J.Y. Pacquet, R. Leroy, US patent $n^{\circ} 6.194 .836$ (Feb. 2001)

${ }^{10}$ P. Jardin et al., Rev. Sci. Instrum. 73 (2002) 789.

${ }^{11}$ F.F. Chen, Introduction to Plasma Physics, 2d edition, Plenum Press, (1984) §1.3.

${ }^{12}$ A. Girard, C. Lécot, K. Serebrennikov, Journal of Computational Physics 191 (2003) 228.

${ }^{13}$ T. Lamy, J.C. Curdy, R. Geller, C. Peaucelle, P. Sole, P. Sortais, T. Thuillier, D. Voulot, in Proceedings of EPAC 2002, Paris, France (2002) 1724.

${ }^{14}$ O. Tuske et al. in : Proc. ICIS03, 10th International Conference on Ion Sources (Dubna, Russia, 2003), RSI, accepted for publication.

${ }^{15}$ H. Tawara and T. Kato, Atomic Data and Nuclear Data Tables 36 (1987) 246

${ }^{16} \mathrm{P}$. Jardin, in preparation 